\title{
EX-SITU CONSERVATION OF THREATENED FOREST TREE SPECIES FOR SUSTAINABLE USE OF FOREST GENETIC RESOURCES IN BANGLADESH
}

\author{
Rahman, M. M., W. Parvin, N. Sultana and S. A. M. Tarek \\ Silviculture Genetics Division, Bangladesh Forest Research Institute, Chittagong-4000
}

\begin{abstract}
The present study was carried out to advance towards the conserving threatened forest tree species of Bangladesh under $e x$-situ conservation system. Four thousand plants of forty eight threatened forest tree species were conserved at three areas, namely Institute of Forestry and Environmental Sciences, Chittagong University; Radar Unit of Bangladesh Air Force, Cox's Bazar; and the Keochia research station of Bangladesh Forest Research Institute. The average $90-95 \%$ plants survived in each conservation site and the maximum average plant height was recorded as $4.0 \mathrm{~m}$ after two years of plantation. It will provide a wider buffer for the protection of the forest gene resources.
\end{abstract}

Key words: Ex-situ conservation, threatened tree species, forest genetic resource, sustainable use, germplasm.

\section{INTRODUCTION}

Bangladesh is located between latitudes $20^{\circ} 34^{\prime}$ and $26^{\circ} 38^{\prime}$ north, and betweenlongitudes $88^{\circ} 01^{\prime}$ and $92^{\circ} 41^{\prime}$ east. The country consists mostly of flood plains $(80 \%)$ with some hilly areas $(12 \%)$, with a subtropical monsoon climate (Islam 2003). In winter, temperature ranges from a minimum of $7^{\circ} \mathrm{C}$ to $13^{\circ} \mathrm{C}$ to a maximum of $23^{\circ}$ to $32^{\circ} \mathrm{C}$. In summer, the temperature varies from $36^{\circ} \mathrm{C}$ to $41^{\circ} \mathrm{C}$. The mean annual rainfall ranges from 143 to $434 \mathrm{~cm}$ (BBS 1994). The population stood at 131.6 million in 2001. Bangladesh has a total area of 14.39 million hectares, of which 9.12 million ha are cultivated, 2.14 million ha public forests, 0.27 million ha village groves and 1.64 million ha constantly under water. The remaining land area (1.22 million hectares) is occupied by tea gardens, uncultivable areas, rural and urban houses and ponds (Kibria et al. 2000). The area covered by government and village forests is about $16 \%$ of the total land area; however, only 0.93 million ha $(6.5 \%)$ is under tree cover, which is about $40 \%$ of the forests controlled by the government. The remaining $60 \%$ include denuded lands (grassland, scrubland and encroached areas). About 24,000 ha of forest are lost annually as a result of homestead development, urbanization and deforestation (Anon 1992). Bangladesh, due to its unique geophysical location and a suitable climatic condition, is exceptionally endowed with a rich variety of biodiversity (Nishat et al. 2002). Nevertheless, in last years, like most other regions of the world, Bangladesh also went through a critical period unsuitable for country's biodiversity and ecosystem. The government, along with various international conservation agencies, is trying to improve and manage this overwhelming situation.

The forests of Bangladesh cover four major vegetation types occurring in three distinctly different ecosystems, i.e. Hill forests (evergreen to semi-evergreen); Plain land sal (Shorea robusta) forests, Mangrove forests, and Village or Homestead forests. Although, once very rich in biodiversity during the last few decades all forest and ecosystems of the country have been heavily degraded (Mukul et al. 2008). There have some contradictions on the actual forest coverage of the country. Although the official forest coverage is 2.53 million ha representing nearly $17.5 \%$ of the country's total land area, only 1.52 million ha of them are under the jurisdiction of the Forest Department (Khan et al. 2007, FAO 2006). In addition to that, most of the forests of the country are geographically located only in few districts and are poorly stocked.

Bangladesh's forests have decreased significantly in terms of both area and health status over the last few decades. The annual deforestation rate is estimated to be around 3.3\% (Khan 2004). The increasing 
population of Bangladesh continues to put pressure on existing forest resources. Present productivity of forest has declined to a range of 1.5-2.5 $\mathrm{m}$ per hectare per annum from 7-8 $\mathrm{m}$ per hectare per annum that was accounted twenty years ago (MoEF 1993). Moreover, canopy closure density, number of trees per hectare and over all plant biodiversity has declined rapidly (FAO 2000). As a consequence, the quality of the forestland as 'wildlife habitat' has also been reduced. Forest cover loss in the country has not been comprehensively studied until now and the quantification of this loss is largely assessed by periodic visual observations.

In Bangladesh, some 2,260 species of plant reported alone from the Chittagong Hill Tracts, which falls between two major floristic regions of Asia (MoEF 1993). Until now, an estimated 5,700 species of angiosperms alone, including 68 woody legumes, 130 fibre yielding plants, 500 medicinal plants, 29 orchids, 3 gymnosperms and 1,700 pteridophytes have been recorded from the country (Firoz et al. 2004). A great number of plants are already extinct from Bangladesh. A reliable statistic on country's plant diversity is still unavailable, nevertheless, it is anticipated that already $10 \%$ of country's plant species have gone extinct. A recent inventory identified 106 vascular plants with risks of various degrees of threats (Khan et al. 2001). However, according to the 'Encyclopedia of Flora and Fauna of Bangladesh' (Volumes 5-12) on vascular plants (Pteridophytes, gymnosperms and angiosperms) about 13\% species were designated as threatened (Siddiqui et al. 2007a, b, Ahmed et al. 2008b,c, Ahmed et al. 2009a,b,c,d). A few families are significantly threatened; for example about 53\% species of Orchidaceae are threatened (94 species out of 179), whereas in Lamiaceae it is more than $30 \%$ (26 species out of 86). Needless to say, these threatened statuses are purely in the national context. The information in the Encyclopedia can be considered as the most recent update for Bangladesh. Among the total 3,813 angiosperm species, 449 species are threatened in their natural habitat. Many species are still being exploited to the extent that they are now listed as vulnerable or endangered with a distinct number of populations threatened with extinction. Conservation is essential for sustainable forest management and the promising potential of forestry to contribute national development objectives.

The main challenges of forest gene conservation and management are related to ongoing forest degradation and encroachment. This is a national scale problem that requires multi-sectoral solutions towards land use planning and improved livelihoods. Forest management is limited by capacity and budgetary constraints. In addition to the ecological, aesthetic and ethical consequences, loss of biodiversity and global warming pose a range of potential threats to human health, nutrition (Chivian and Bernstein 2008) and economics as a whole (TEEB 2010). Considering the above points, Silviculture Genetics Division of the BFRI has attempted to make a plan for studying the conservation of the threatened forest species under $e x$-situ condition.

\section{Planting materials}

\section{MATERIAL AND METHODS}

The seedlings of 48 threatened forest tree species were used for conservation programme. The seedlings were developed through different propagation methods and maintained at the nursery of Silviculture Genetics Division of BFRI, Chittagong. Seeds, branch cuttings, young twigs, branch shoot tips were used to develop seedlings through macro propagation under greenhouse and nursery condition whereas seeds and vegetative parts were used for micro-propagation in tissue culture laboratory. Three areas namely the Keochia, Research Station of BFRI, Institute of Forestry and Environmental Sciences (IFESCU), Chittagong University (CU), and the Radar unit of Bangladesh Air Force (BAF), Cox's Bazar were selected for conservation. Combined efforts were made among BFRI, IFESCU, and BAF, Cox's Bazar radar unit to raise and maintain the plantation. IUCN Red List categories and criteria of 1994 were apparently followed to determine threatened vascular plant species in Bangladesh. 


\section{Propagules development}

Seed germination, seedling raising and the practice of vegetative propagation methods were emphasized to produce plant propagules.

\section{Seed germination and seedling rising}

Seeds were collected based on the flowering season of the threatened plant species through exploration of different natural habitat like the forests of Chittagong and Chittagong Hill Tracts (CHTs), Sylhet, Dhaka and Cox's Bazar. Seeds were germinated in seed beds at mist house and direct sowing in the polybags of Silviculture Genetics Division nursery of BFRI. The seedlings were managed up to one year in the nursery and planted in the conservation site with a spacing of 6 ' $\times 6$ '.

\section{Propagules development through vegetative propagation}

\section{Propagation by stem cuttings}

Some of the species were propagated through vegetative means by stem cuttings. Due to the availability of propagation materials, season of the year or the facilities available in the nursery, it was easy to develop root and shoot of cutting base. The cuttings were taken from the ends of the young twigs or the top of the plant. This type of cutting involves a piece of the stem plus about 3 to 4 leaves. Sometimes the tip cuttings were also used. Each piece of stem should be from 2 to 5 inches long.

Different rooting media were used for root and shoot induction at the base of cuttings; these were $100 \%$ sand, $100 \%$ garden soil, $100 \%$ sphagnum peat moss, $50 \%$ coarse sand plus and 50\% sphagnum peat moss. The poly bag and plastic pots $\left(5^{\prime \prime} \times 7^{\prime \prime}\right)$ were used as containers. After filling the containers with rooting medium and wetted these thoroughly before inserting the cuttings. The medium should be kept moist until rooting occurs. Synthetic rooting hormones Indole-3-butyric acid (IBA)and $\alpha$ naphthalene acetic acid (NAA) with different concentrations (100 ppm, 200 ppm, 300 ppm, 400 ppm and $500 \mathrm{ppm}$ ) were used to develop root at the cutting base. The cutting base was depped into different concentrations of rooting hormones exposed for certain time, such as 10, 20, 40, and 60 minutes. The experiments were carried out under greenhouse condition to provide maximum humidity $90-100 \%$ and temperature $29^{\circ} \mathrm{C}$. Depending on the type of plant species it takes 2-3 months for rooting. When the cuttings have grown roots, they were one-half inch or more in length. It was potted in a more permanent mixture of garden soil and cow dung 3: 1 and allowed them to grow under nursery condition.

\section{Propagation through tissue culture}

Development of propagules for mass propagation and conservation of threatened tree species have been done in tissue culture laboratory of Silviculture Genetics Division of BFRI since 1990. The seedlings of some of the important species like as Haldu (Adina cordifolia) were produced through tissue culture technique. The TC seedlings were also used as propagule materials in the conservation sites.

\section{Statistical analysis}

All experiments were performed as Completely Randomized Design (CRD). Data were analyzed using statistical analysis system (SAS v 9.3) and means were statistically compared using LSD test. The significance level was set up at $\mathrm{p}<0.05$.

\section{RESULTS AND DISCUSSION}

Silviculture Genetics Division of BFRI has been conducting research study on conservation and centralization of threatened forest tree species for their sustainable management since 2010. Accordingly, it has been developed the propagation methods for different species to maximize the production of plant propagules. According to the records of the Red Data Book there are 106 species of 
46 families of the vascular plants of Bangladesh. Among them 48 species of 32 families were conserved at three protected areas of Bangladesh which are Keochia research station, Chittagong of BFRI, IFESCU of Chittagong University, and the Radar unit of Bangladesh Air Forces, Cox's Bazar (Table 1).

The plant species were categories as the IUCN Red List categories (www.Iucnredlist.org) near threatened, vulnerable, endangered, critically endangered and regionally extinct in the habitat. The most widely distributed family is represented by Sterculiaceae (5 species). The species were mostly distributed in the evergreen, semi evergreen and deciduous forests of Chittagong, Chittagong Hill tracts, Cox's Bazar and Sylhet district of Bangladesh.

Table1. List of threatened plant species conserved at IFESCU, Chittagong University, Radar Unit of Bangladesh Air force, Cox's Bazar and Keochia Research Station of BFRI.

\begin{tabular}{|c|c|c|c|c|c|c|}
\hline $\begin{array}{l}\text { Local } \\
\text { name }\end{array}$ & $\begin{array}{c}\text { Scientific } \\
\text { name }\end{array}$ & Family & $\begin{array}{l}\text { Natural habitat } \\
\text { (forests) }\end{array}$ & Tree features & $\begin{array}{l}\text { Current } \\
\text { status }\end{array}$ & Conservation site \\
\hline Asok & $\begin{array}{l}\text { Saraca } \\
\text { asoca }\end{array}$ & Caesalpiniaceae & $\begin{array}{l}\text { Chittagong and } \\
\text { CHTs }\end{array}$ & $\begin{array}{l}\text { Medium (20- 25m } \\
\text { height) } \\
\text { Evergreen }\end{array}$ & Vulnerable & $\begin{array}{l}\text { IFESCU, Radar unit, } \\
\text { Keochia, BFRI }\end{array}$ \\
\hline Bandorhola & $\begin{array}{l}\text { Duabanga } \\
\text { grandiflora }\end{array}$ & Sonneratiaceae & $\begin{array}{l}\text { Chittagong, Cox's } \\
\text { Bazar and Sylhet }\end{array}$ & $\begin{array}{l}\text { Large }(25-40 \mathrm{~m}), \\
\text { Deciduous }\end{array}$ & Endangered & Radar unit \\
\hline Banshpata & $\begin{array}{l}\text { Podocarpus } \\
\text { neriifolius }\end{array}$ & Podocarpaceae & $\begin{array}{l}\text { Chittagong and } \\
\text { Cox’s Bazar }\end{array}$ & Large, Evergreen & $\begin{array}{l}\text { Critically } \\
\text { endangered }\end{array}$ & IFESCU \\
\hline Barela & $\begin{array}{l}\text { Holigarna } \\
\text { caustica }\end{array}$ & Anacardiaceae & $\begin{array}{l}\text { Chittagong, CHTs, } \\
\text { Sylhet, and Cox's } \\
\text { Bazar }\end{array}$ & Large, Evergreen & Vulnerable & $\begin{array}{l}\text { IFESCU, Radar } \\
\text { unit }\end{array}$ \\
\hline Bariakat & $\begin{array}{l}\text { Berrya } \\
\text { cordifolia }\end{array}$ & Malvaceae & Chittagong & Large, Deciduous & Vulnerable & $\begin{array}{l}\text { IFESCU, Radar } \\
\text { unit }\end{array}$ \\
\hline Barun & $\begin{array}{l}\text { Crataeva } \\
\text { magna }\end{array}$ & Capparaceae & $\begin{array}{l}\text { Chittagong and } \\
\text { CHTs }\end{array}$ & Small to medium & Vulnerable & $\begin{array}{l}\text { IFESCU, Radar } \\
\text { unit }\end{array}$ \\
\hline Batna & $\begin{array}{l}\text { Castanopsis } \\
\text { indica }\end{array}$ & Fagaceae & $\begin{array}{l}\text { Chittagong, CHTs, } \\
\text { Sylhet }\end{array}$ & Medium to large & Vulnerable & $\begin{array}{l}\text { IFESCU, Radar } \\
\text { unit }\end{array}$ \\
\hline Bazna & $\begin{array}{l}\text { Zanthoxylum } \\
\text { rhetsa }\end{array}$ & Rutaceae & $\begin{array}{l}\text { Chittagong, CHTs, } \\
\text { Sylhet }\end{array}$ & $\begin{array}{l}\text { Medium, } \\
\text { Deciduous }\end{array}$ & Endangered & $\begin{array}{l}\text { IFESCU, Radar } \\
\text { unit }\end{array}$ \\
\hline Bohera & $\begin{array}{l}\text { Terminalia } \\
\text { bellirica }\end{array}$ & Combretaceae & $\begin{array}{l}\text { Chittagong, CHTs, } \\
\text { Cox's Bazar and } \\
\text { Sylhet }\end{array}$ & Large, Deciduous & Vulnerable & IFESCU \\
\hline Boilam & $\begin{array}{l}\text { Anisoptera } \\
\text { scaphula }\end{array}$ & Depterocarpaceae & $\begin{array}{l}\text { Chittagong and } \\
\text { Cox'sBazaar }\end{array}$ & Large, Evergreen & $\begin{array}{l}\text { Critically } \\
\text { endangered }\end{array}$ & IFESCU \\
\hline Boxbadam & $\begin{array}{l}\text { Sterculia } \\
\text { foetida }\end{array}$ & Sterculiaceae & Chittagong & Large, Evergreen & $\begin{array}{l}\text { Vulnerable / } \\
\text { Endangered }\end{array}$ & $\begin{array}{l}\text { IFESCU, Radar } \\
\text { unit }\end{array}$ \\
\hline Buddhunarkili & $\begin{array}{l}\text { Pterygota } \\
\text { alata }\end{array}$ & Sterculiaceae & $\begin{array}{l}\text { Chittagong, Cox's } \\
\text { Bazar }\end{array}$ & Large, Deciduous & Endangered & $\begin{array}{l}\text { IFESCU, Radar unit, } \\
\text { Keochia, BFRI }\end{array}$ \\
\hline Chalmugra & $\begin{array}{l}\text { Hydnocarpus } \\
\text { kurzii }\end{array}$ & Flacourtiaceae & $\begin{array}{l}\text { Chittagong, Cox's } \\
\text { Bazar and Sylhet }\end{array}$ & $\begin{array}{l}\text { Medium, } \\
\text { Evergreen }\end{array}$ & $\begin{array}{l}\text { Critically } \\
\text { endanger }\end{array}$ & IFESCU \\
\hline Champa & $\begin{array}{l}\text { Magnolia } \\
\text { champaca }\end{array}$ & Magnoliaceae & $\begin{array}{l}\text { Chittagong, CHTs, } \\
\text { Sal forest and } \\
\text { Sylhet }\end{array}$ & $\begin{array}{l}\text { Medium, } \\
\text { Evergreen }\end{array}$ & Vulnerable & Radar unit \\
\hline Chatian & $\begin{array}{l}\text { Alstonia } \\
\text { scholaris }\end{array}$ & Apocynaceae & $\begin{array}{l}\text { Chittagong, CHT } \\
\text { and Cox's Bazar }\end{array}$ & $\begin{array}{l}\text { Medium to large, } \\
\text { Evergreen }\end{array}$ & Endangered & $\begin{array}{l}\text { IFESCU, Keochia, } \\
\text { BFRI }\end{array}$ \\
\hline Civit & $\begin{array}{l}\text { Swintonia } \\
\text { floribunda }\end{array}$ & Anacardiaceae & $\begin{array}{l}\text { Chittagong, CHTs } \\
\text { and Cox's Bazar }\end{array}$ & Large, Evergreen & Vulnerable & $\begin{array}{l}\text { IFESCU, Radar } \\
\text { unit, Cox's Bazar }\end{array}$ \\
\hline Dharmara & $\begin{array}{l}\text { Stereosperm } \\
\text { um colais }\end{array}$ & Bignoniaceae & $\begin{array}{l}\text { Chittagong, Cox's } \\
\text { Bazar and Sylhet }\end{array}$ & $\begin{array}{l}\text { Medium, } \\
\text { Deciduous }\end{array}$ & $\begin{array}{l}\text { Critically } \\
\text { endangered }\end{array}$ & $\begin{array}{l}\text { IFESCU, Radar } \\
\text { unit }\end{array}$ \\
\hline Dhup & Canarium & Burseraceae & Chittagong, Cox's & Large, Evergreen & Protected & IFESCU \\
\hline
\end{tabular}




\begin{tabular}{|c|c|c|c|c|c|c|}
\hline & resiniferum & & Bazar and Sylhet & & plant & \\
\hline Gutguttya & $\begin{array}{l}\text { Protium } \\
\text { serratum } \\
\text { Serra }\end{array}$ & Burseraceae & $\begin{array}{l}\text { Chittagong, Cox's } \\
\text { Bazar and Sylhet }\end{array}$ & Large, Evergreen & Endangered & IFESCU, Radar unit \\
\hline Haldu & $\begin{array}{l}\text { Adina } \\
\text { cordifolia }\end{array}$ & Rubiaceae & $\begin{array}{l}\text { Chittagong, Cox's } \\
\text { Bazar and Sylhet }\end{array}$ & Large, Deciduous & Endangered & IFESCU \\
\hline Horitoki & $\begin{array}{l}\text { Terminalia } \\
\text { chebula }\end{array}$ & Combretaceae & $\begin{array}{l}\text { Chittagong, CHTs, } \\
\text { Cox's Bazar, Sal } \\
\text { forest and Sylhet }\end{array}$ & $\begin{array}{l}\text { Medium, } \\
\text { Deciduous }\end{array}$ & $\begin{array}{l}\text { Endangered } \\
\text { Vulnerable }\end{array}$ & IFESCU \\
\hline Kalomenda & $\begin{array}{l}\text { Litsea } \\
\text { glutinosa }\end{array}$ & Lauraceae & $\begin{array}{l}\text { Chittagong, CHTs, } \\
\text { Sal forest, Cox's } \\
\text { Bazar and Sylhet }\end{array}$ & $\begin{array}{l}\text { Medium, } \\
\text { Evergreen }\end{array}$ & Vulnerable & Radar unit \\
\hline Kanak & $\begin{array}{l}\text { Schima } \\
\text { wallichii }\end{array}$ & Theaceae & $\begin{array}{l}\text { Chittagong Hill } \\
\text { forest, Cox's Bazar } \\
\text { and Sylhet }\end{array}$ & $\begin{array}{l}\text { Large size } \\
\text { Evergreen }\end{array}$ & Endangered & $\begin{array}{ll}\text { IFESCU, Radar } \\
\text { unit }\end{array}$ \\
\hline Kannyari & $\begin{array}{l}\text { Gardenia } \\
\text { coronaria }\end{array}$ & Rubaceae & $\begin{array}{l}\text { Chittagong, Cox's } \\
\text { Bazar and Sylhet. }\end{array}$ & $\begin{array}{l}\text { Small to medium } \\
(15-20 \mathrm{~m})\end{array}$ & Vulnerable & IFESCU \\
\hline Karanja & $\begin{array}{l}\text { Pongamia } \\
\text { Pinnata }\end{array}$ & Fabaceae & $\begin{array}{l}\text { Chittagong, CHTs, } \\
\text { Cox's Bazar, Sylhet } \\
\text { and Sundarbans }\end{array}$ & Small, Evergreen & Vulnerable & $\begin{array}{l}\text { IFESCU, Radar } \\
\text { unit }\end{array}$ \\
\hline Keron & $\begin{array}{l}\text { Pongamiapi } \\
\text { nnata }\end{array}$ & Fabaceae & $\begin{array}{l}\text { Chittagong, CHTs } \\
\text { Sylhet, Sundarbans } \\
\text { and Sal forest. }\end{array}$ & $\begin{array}{l}\text { Medium, } \\
\text { Evergreen }\end{array}$ & Vulnerable & $\begin{array}{l}\text { IFESCU, Radar } \\
\text { unit }\end{array}$ \\
\hline Knema & $\begin{array}{l}\text { Knema } \\
\text { bengaliensis }\end{array}$ & Myristicaceae & $\begin{array}{l}\text { Chittagong and } \\
\text { Cox's Bazar }\end{array}$ & $\begin{array}{l}\text { Medium, } \\
\text { Evergreen }\end{array}$ & $\begin{array}{l}\text { Rare/ } \\
\text { endangered }\end{array}$ & $\begin{array}{l}\text { IFESCU, CU; Radar } \\
\text { unit, Cox's Bazar }\end{array}$ \\
\hline Kuchila & $\begin{array}{l}\text { Strychnosnux } \\
\text {-vomica }\end{array}$ & Loganiaceae & $\begin{array}{l}\text { Chittagong and } \\
\text { Cox's Bazar }\end{array}$ & Small, Deciduous & Vulnerable & IFESCU, Radar unit, \\
\hline Kushum & $\begin{array}{l}\text { Schleichera } \\
\text { oleosa }\end{array}$ & Sapindaceae & $\begin{array}{l}\text { Chittagong, Cox's } \\
\text { Bazar and Sylhet }\end{array}$ & Large, Deciduous & Endangered & IFESCU \\
\hline Matang & $\begin{array}{l}\text { Carallia } \\
\text { brachiata }\end{array}$ & Rhizophoraceae & $\begin{array}{l}\text { Chittagong, CHTs } \\
\text { and Sylhet }\end{array}$ & $\begin{array}{l}\text { Medium, } \\
\text { Deciduous }\end{array}$ & Vulnerable & IFESCU \\
\hline Minjiri & $\begin{array}{l}\text { Cassia } \\
\text { siamea }\end{array}$ & Caesalpiniaceae & $\begin{array}{l}\text { Chittagong and } \\
\text { CHTs }\end{array}$ & $\begin{array}{l}\text { Medium to large, } \\
\text { Evergreen or } \\
\text { Semi-deciduous }\end{array}$ & Vulnerable & IFESCU \\
\hline Mohua & $\begin{array}{l}\text { Madhuca } \\
\text { longifolia }\end{array}$ & Sapotaceae & Sal forest & $\begin{array}{l}\text { Medium, } \\
\text { Deciduous }\end{array}$ & Vulnerable & $\begin{array}{l}\text { Keochia, BFRI. } \\
\text { Radar unit }\end{array}$ \\
\hline Moos & $\begin{array}{l}\text { Brownlowia } \\
\text { elata }\end{array}$ & Tiliaceae & $\begin{array}{l}\text { Chittagong and } \\
\text { Cox's Bazar }\end{array}$ & $\begin{array}{l}\text { Medium, Semi- } \\
\text { evergreen }\end{array}$ & Vulnerable & IFESCU, Radar unit \\
\hline Motor korai & $\begin{array}{l}\text { Albizia } \\
\text { lucidior }\end{array}$ & Mimosaceae & $\begin{array}{l}\text { Chittagong, CHTs, } \\
\text { Sal forest and Sylhet }\end{array}$ & Medium & Endangered & IFESCU \\
\hline Muskundo & $\begin{array}{l}\text { Pterospermum } \\
\text { acerifolium }\end{array}$ & Sterculiaceae & $\begin{array}{l}\text { Chittagong, CHTs, } \\
\text { Cox's Bazar and Sylhet }\end{array}$ & Large, Evergreen & Vulnerable & IFESCU \\
\hline Padok & $\begin{array}{l}\text { Pterocarpus } \\
\text { indicus }\end{array}$ & Fabaceae & Chittagong & Large, Deciduous & $\begin{array}{l}\text { Critically } \\
\text { endangered }\end{array}$ & IFESCU \\
\hline Parul & $\begin{array}{l}\text { Stereosperm } \\
\text { umsuaveolens }\end{array}$ & Bignoniaceae & $\begin{array}{l}\text { Chittagong and } \\
\text { CHTs }\end{array}$ & $\begin{array}{l}\text { Medium, } \\
\text { Deciduous }\end{array}$ & Endangered & IFESCU \\
\hline Patagota & $\begin{array}{l}\text { Firmiana } \\
\text { colorata }\end{array}$ & Sterculiaceae & $\begin{array}{l}\text { Chittagong, CHTs } \\
\text { and Cox's Bazar }\end{array}$ & $\begin{array}{l}\text { Medium, } \\
\text { Deciduous }\end{array}$ & Endangered & IFESCU, Radar unit, \\
\hline Rakton & $\begin{array}{l}\text { Lophopetalum } \\
\text { wightianum }\end{array}$ & Celastraceae & $\begin{array}{l}\text { Chittagong, CHTs } \\
\text { Cox's Bazar and Sylhet }\end{array}$ & Large, Evergreen & Endangered & IFESCU \\
\hline $\begin{array}{l}\text { Rokto } \\
\text { chandon }\end{array}$ & $\begin{array}{l}\text { Adenanthera } \\
\text { pavonina }\end{array}$ & Mimosaceae & $\begin{array}{l}\text { Chittagong, CHTs } \\
\text { and Sylhet }\end{array}$ & Medium & Endangered & IFESCU \\
\hline Tabevuiya & $\begin{array}{l}\text { Tabebuia } \\
\text { rosea }\end{array}$ & Bignoniaceae & $\begin{array}{l}\text { Chittagong, CHTs } \\
\text { and Sylhet }\end{array}$ & Large, Deciduous & Endangered & IFESCU \\
\hline Tali & Palaquium & Sapotaceae & Chittagong, Cox's & Medium to large, & Protected & IFESCU \\
\hline
\end{tabular}




\begin{tabular}{|l|l|l|l|l|l|l|}
\hline & polyanthum & & Bazar and Sylhet & Evergreen & plant & \\
\hline Tamal & $\begin{array}{l}\text { Diospyros } \\
\text { montana }\end{array}$ & Ebenaceae & $\begin{array}{l}\text { Chittagong and } \\
\text { CHTs }\end{array}$ & $\begin{array}{l}\text { Medium, } \\
\text { Deciduous }\end{array}$ & Vulnerable & IFESCU, Radar unit \\
\hline Titpai & $\begin{array}{l}\text { Millettia } \\
\text { peguensis }\end{array}$ & Elaeocarpaceae & $\begin{array}{l}\text { Chittagong, Cox's } \\
\text { Bazar, Sal forest, } \\
\text { and Sylhet }\end{array}$ & $\begin{array}{l}\text { Medium to large, } \\
\text { Deciduous }\end{array}$ & Endangered & IFESCU \\
\hline Toon & $\begin{array}{l}\text { Toona } \\
\text { ciliata }\end{array}$ & Meliaceae & $\begin{array}{l}\text { Chittagong, Cox's } \\
\text { Bazar, Sal forest and } \\
\text { Sylhet }\end{array}$ & $\begin{array}{l}\text { Medium, Mixed } \\
\text { evergreen }\end{array}$ & Vulnerable & IFESCU \\
\hline Udal & $\begin{array}{l}\text { Sterculia } \\
\text { villosa }\end{array}$ & Sterculiaceae & $\begin{array}{l}\text { Chittagong, Cox's } \\
\text { Bazar and Sylhet }\end{array}$ & $\begin{array}{l}\text { Medium (15-20 m), } \\
\text { Evergreen. }\end{array}$ & Endangered & IFESCU \\
\hline Uriaam & $\begin{array}{l}\text { Mangifera } \\
\text { sylvatica }\end{array}$ & Anacardiaceae & $\begin{array}{l}\text { Chittagong, Cox's } \\
\text { Bazar and Sylhet }\end{array}$ & Large, Evergreen & $\begin{array}{l}\text { Critically } \\
\text { endangered }\end{array}$ & IFESCU \\
\hline Vuikodom & $\begin{array}{l}\text { Hymenodicty } \\
\text { lonorixensis }\end{array}$ & Rubiaceae & $\begin{array}{l}\text { Chittagong Hills, } \\
\text { CHT and Cox's } \\
\text { Bazar }\end{array}$ & $\begin{array}{l}\text { Medium to large, } \\
\text { Evergreen }\end{array}$ & Vulnerable & $\begin{array}{l}\text { IFESCU, Radar } \\
\text { unit }\end{array}$ \\
\hline
\end{tabular}

Total 5,632 plants of 48 species were conserved under the three conservation sites. The species and their distribution in new habitat are shown in Table 1 and Fig. 1. The planting materials were developed through seed collection and seedling rising, stem cutting and tissue culture as well. The results revealed that seed germination were enhanced in the seed beds under mist house condition rather than direct sowing in polybags. However, the rooting percentage and cutting survivality were achieved $80-90 \%$ of Adina cordifolia in rooting media having 50\% coarse sand and 50\% sphagnum peat moss with 40 minutes treated with $500 \mathrm{ppm}$ IBA. On the other hand, in vitro multiple shoots of $A$. cordifolia were developed in MS medium supplemented with $(1.0 \mathrm{mg} / \mathrm{L} \mathrm{BAP}+0.5 \mathrm{mg} / \mathrm{L} \mathrm{NAA})$. The shoots were rooted in $1 / 2 \mathrm{MS}$ medium supplemented with $1.0 \mathrm{mg} / \mathrm{L}$ IBA. The plant propagules were maintained in the nursery for their growth up to the planting height. The first demonstration plots were raised in 2014 with the collaboration of IFESCU at Chittagong University. In 2015, plantation raised at radar unit of Bangladesh Air Forces, Cox's Bazar and the Keochia research station of BFRI.

The field performance of all conserved species was found promising both in survival and growth rate in different conservation sites. About 90-95\% plants survived in each conservation site (Table 2 and Fig.2).

Table 2. Survival percentage of conserved threatened tree species at three conservation sites.

\begin{tabular}{|c|c|c|c|}
\hline Conservation Sites & No. of species & No. of seedlings & Survival \% \\
\hline IFESCU Campus, CU & 44 of 48 & 4,000 no. & $95 \pm 1.5^{*}$ \\
\hline $\begin{array}{l}\text { Radar unit, Bangladesh Air } \\
\text { Force, Cox's Bazar }\end{array}$ & 24 of 48 & 1,282 no. & $92.33 \pm 0.76^{*}$ \\
\hline $\begin{array}{l}\text { Keochia research station, } \\
\text { BFRI. }\end{array}$ & 4 of 48 & 350 no. & $90.66 \pm 0.76^{*}$ \\
\hline
\end{tabular}

*values indicate the standard error of mean

The growth and field performance of some species are shown in Fig. 1 and Fig. 2 at the conservation site of IFESCU of Chittagong University. The maximum plant height was recorded $2.91 \mathrm{~m}$ for the species Bhuddunarkili (Pterygota alata) and the diameter $0.11 \mathrm{~m}$ for the species keron (Pongamia pinnata) after two years of plantation (Fig 1). 


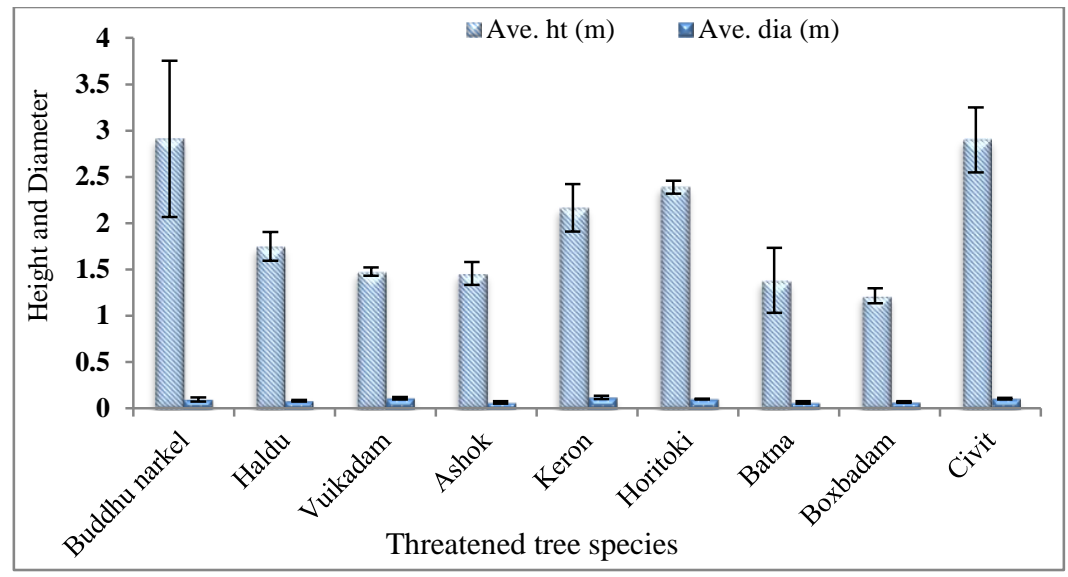

Fig. 1. Growth performance of different species at IFESCU, Chittagong University campus after two years of plantation.

The values of forest gene conservation are equally important in meeting the long term interests of Bangladesh and its future generations. These include environmental protection and the conservation of biodiversity and natural heritage. Therefore, implementation of the National Forest Gene Conservation Strategy ensures that seed and planting materials of different populations of desired tree species will be available when a planting need arises in the country. Under these circumstances, plants protected ex situ, for instance in protected areas, are increasingly important to supplement in situ conservation (Smith et al. 2003, Sarasan et al. 2006, Engelmann et al. 2007, Li and Pritchard 2009).

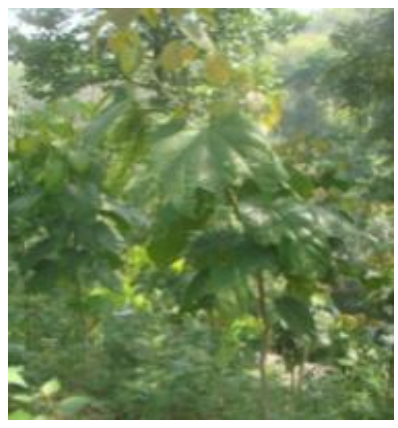

a

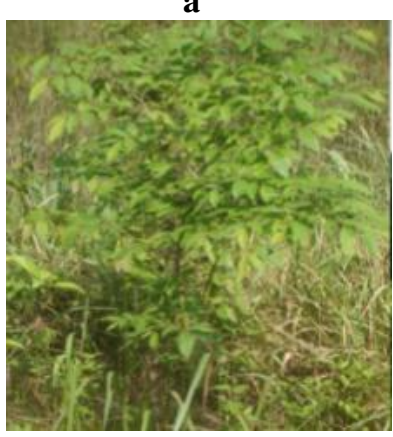

e

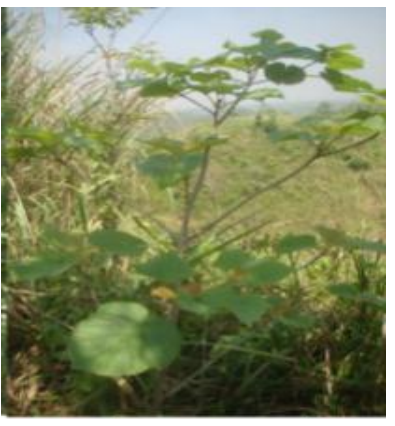

b

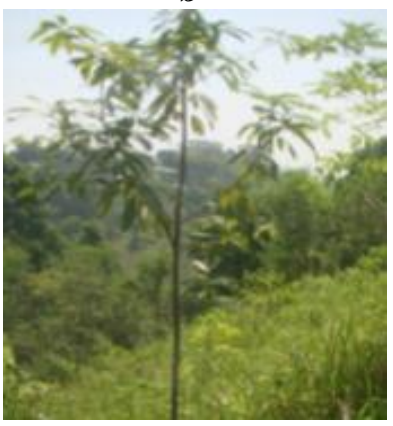

$\mathbf{f}$

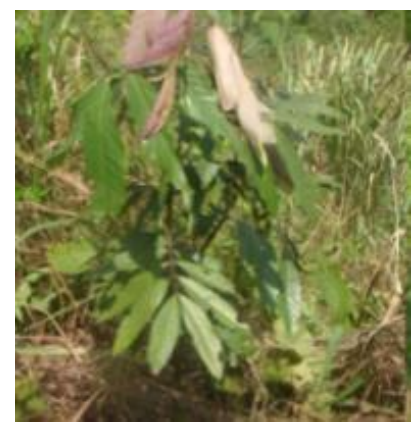

c

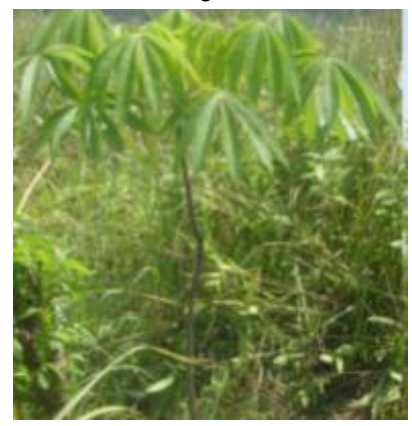

g

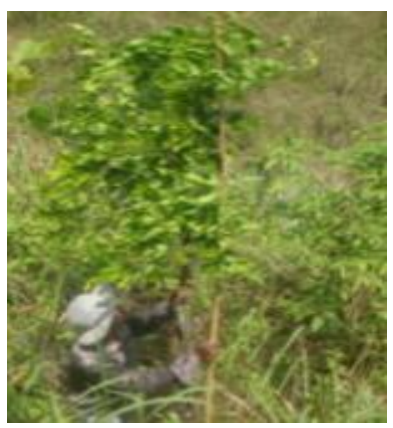

d

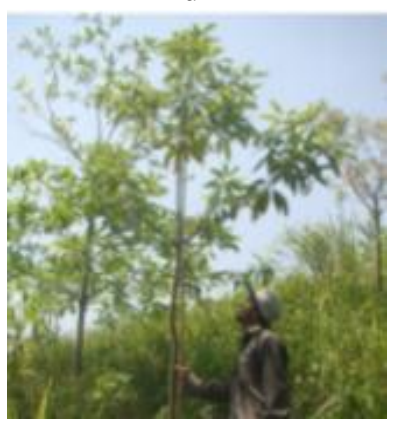

h

Fig. 2. Conservation plot of different plant species at IFESCU, Chittagong University campus: a. Buddha narkel; b. Haldu; c. Asok; d. Keron; e. Haritoki; f. Batna; g. Boxbadam; and h. Civit.

From ex situ collections, conserved in the form of living plants, stored seeds and tissue cultures, plants can be reintroduced to their original or, where necessary, eco-logically restored habitats (Cochrane et al. 2007, Guerrant and Kaye 2007). Alternatively, new areas considered to provide more 
favorable living conditions, as climate change proceeds, can be targeted (McLachlan et al. 2007, Richardson et al. 2009). Conservation is essential for sustainable forest management and the promising potential of forestry to contribute to national development objectives, such as poverty alleviation and socio-economic development has been realized.

The main challenges of forest gene conservation and management are related to ongoing forest degradation and encroachment. This is a national scale problem that requires multi-sectoral solutions towards land use planning and improved livelihoods. Despite a rapid loss and degradation of wild habitats, biodiversity conservation has received a wider attention in Bangladesh in the present years (Mukul et al. 2017, Mukul 2007). The country has also adopted various in situ and ex situ conservation measures to maintain its rich biological heritage. Declarations of protected areas, ecologically critical areas, World Heritage Site, Ramsar Sites are among the widely used ways for in situ conservation (Mukul et al. 2008). At present, the country has 38 protected areas including 17 national parks and 21 wildlife sanctuaries distributed across the country. Together, the protected areas of Bangladesh cover nearly $17.5 \%$ of the forest area and $1.8 \%$ of country's total land area (Mukul et al. 2017, Mukul et al. 2008). In addition to that, the country has seven eco-parks, two safari parks and botanical gardens which also contribute significantly to the conservation of country's dwindling biodiversity. However the three conservation sites could be a living germplasm center with a greater gene pool of 48 threatened tree species of Bangladesh.

The conservation of threatened forest tree species can be achieved through an integrated approach balancing in situ (conservation in natural habitats) and ex situ (conservation away from the natural habitats) strategies. In situ conservation offers the advantages of allowing natural selection to continue. However, when habitat destruction is inevitable, endangered species need to be preserved by ex situ means before they become extinct. Ex situ conservation can also provide the opportunity to study the biology of endangered species in order to eventually consider successful species recovery programs like restoration and reintroduction. It also has the advantage of preserving plant material and making it available for research purposes, without damaging the natural populations. Ex situ is therefore complementary to in situ conservation and can act as an "insurance policy" when species are threatened in their natural habitats.

\section{ACKNOWLEDGEMENTS}

The authors cordially acknowledge the Ministry of Environment, Forest and Climate Change as well as the Director of Bangladesh Forest Research Institute for the financial and logistic support to carry out the study.

\section{REFERENCES}

Ahmed, Z. U., Z. N. T. Begum, M. A. Hassan, M. Khondker, S. M. H. Kabir, M. Ahmad, A. T. A. Ahmed, A. K. A. Rahman and E. U. Haque (eds). 2008b. Encyclopedia of Flora and Fauna of Bangladesh. Angiosperms: Dicotyledons (Acanthaceae-Asteraceae). Vol. 6. Asiatic Society of Bangladesh, Dhaka, pp. 1-408.

Ahmed, Z. U., M. A. Hassan, Z. N. T. Begum, M. Khondker, S. M. H. Kabir, M. Ahmad, A. T. A. Ahmed, A. K. A. Rahman, and E. U. Haque (eds). 2008c. Encyclopedia of Flora and Fauna of Bangladesh. Angiosperms: Monocotyledons (Orchidaceae-Zingiberaceae). Vol. 12. Asiatic Society of Bangladesh, Dhaka, pp. 1-552.

Ahmed, Z. U., M. A. Hassan, Z. N. T. Begum, M. Khondker, S. M. H. Kabir, M. Ahmad, A. T. A. Ahmed, A. K. A. Rahman, and E. U. Haque (eds). 2009a. Encyclopedia of Flora and Fauna of Bangladesh. 
Angiosperms: Dicotyledons (Balsaminaceae-Euphorbiaceae). Vol. 7. Asiatic Society of Bangladesh, Dhaka, pp. 1-546.

Ahmed, Z. U., M. A. Hassan, Z. N. T. Begum, M. Khondker, S. M. H. Kabir, M. Ahmad, A. T. A. Ahmed, A. K. A. Rahman, and E. U. Haque (eds). 2009b. Encyclopedia of Flora and Fauna of Bangladesh. Angiosperms: Dicotyledons (Fabaceae-Lythraceae). Vol. 8. Asiatic Society of Bangladesh, Dhaka, pp. $1-478$.

Ahmed, Z. U., M. A. Hassan, Z. N. T. Begum, M. Khondker, S. M. H. Kabir, M. Ahmad, A. T. A. Ahmed, A. K. A. Rahman, and E.U. Haque (eds). 2009c. Encyclopedia of Flora and Fauna of Bangladesh. Angiosperms: Dicotyledons (Magnoliaceae-Punicaceae). Vol. 9. Asiatic Society of Bangladesh, Dhaka, pp. 1-488.

Ahmed, Z. U., M. A. Hassan, Z. N. T. Begum, M. Khondker, S. M. H. Kabir, M. Ahmad, A. T. A. Ahmed, A. K. A. Rahman and E. U. Haque (eds). 2009d. Encyclopedia of Flora and Fauna of Bangladesh. Angiosperms: Dicotyledons (Ranunculaceae-Zygophyllaceae). Vol. 10. Asiatic Society of Bangladesh, Dhaka, pp. 1-580.

Anon. 1992. Forestry Master Plan (FMP) of Bangladesh. Ministry of Environment and Forest, Government of Bangladesh.

BBS. 1994. Statistical Year Book of Bangladesh. 12th ed. Bangladesh Statistics Division, Ministry of Planning, Dhaka. 628 pp.

Chivian, E. and A. Bernstein (eds). 2008. Sustaining life: how human health depends on biodiversity. Oxford University Press, New York. 542 pp.

Cochrane, J. A., A. D. Crawford and L. T. Monks. 2007. The significance of ex situ conservation to reintroduction of threatened plants. Aust. J. Bot. 55: 356-361.

Engelmann, F., M. E. Dulloo, C. Astorga, S. Dussertand and F. Anthony (eds). 2007. Complementary strategies for ex situ conservation of coffee (Coffeaarabica L.) genetic resources. A case study in CATIE, Costa Rica. Topical reviews in Agricultural Biodiversity. Bioversity International, Rome.

FAO. 2000. Forest Resources of Bangladesh. Country Report, Rome. 91 pp.

FAO. 2006. Better forestry, less poverty: A practitioner's guide. 65 pp.

Firoz, R., S. M. Mobasher, M. Waliuzzaman and M. K. Alam (eds). 2004. Proceedings of the Regional Workshops on National Biodiversity Strategy and Action Plan. IUCN Bangladesh Country Office. Dhaka. pp. 1-167.

Guerrant, E. O. and J. T. N. Kaye. 2007. Reintroduction of rare and endangered plants: common factors, questions and approaches. Aust. J. Bot. 55: 362-370.

Islam, S. S. 2003. State of forest genetic resources conservation and management in Bangladesh. Forest Genetic Resources Working Papers. Forest Resources Division, FAO. Rome, Italy. 28 pp.

Khan, M. S., M. M. Rahman and M. A. Ali. 2001. Red Data Book of Vascular Plants of Bangladesh. Bangladesh National Herbarium. Dhaka, pp. 1-179.

Khan, M. A. S. A., M. B. Uddin, M. S. Uddin, M. S. H. Chowdhury and S. A. Mukul. 2007. Distribution and Status of Forests in the Tropic: Bangladesh Perspective. Proc. Pakistan Acad. Sci. 44: 145-153.

Khan N. A., J. K. Choudhury and K. S. Huda. 2004. Forestry Sector Review Report. Bangladesh Forest Department, Ministry of Environment and Forest, Dhaka, Bangladesh. 
Kibria, M.G., D. C. Sarker, M. A. T. Hossain, M. A. Mannan, M. A. Motaleband S. S Islam. 2000. Forest Statistics of Bangladesh, Bulletin 4. Forest Economics Division, Bangladesh Forest Research Institute, Chittagong. 119 pp.

Li, D. Z. and H. W. Pritchard. 2009. The science and economics of ex-situ plant conservation. Trends Plant Sci. 14: 614-621.

McLachlan, J. S., J. J. Hellman and M. W. Schwartz. 2007. A framework for debate of assisted migration in an era of climate change. Conserv. Biol. 21: 297-302.

MoEF. 1993. Forestry Master Plan-Main Report. ADB (TA No. 1355-BAN), UNDP/FAO BGD 88/025. Ministry of Environment and Forest (MoEF), Dhaka.

Mukul, S. A., M. B. Uddin, M. S. Uddin, M. A. S. A. Khan and B. Marzan. 2008. Protected areas of Bangladesh: current status and efficacy for biodiversity conservation. Proc. Pakistan Acad. Sci. 45: 59-68.

Mukul, S. A., A. Z. M. M. Rashid and N. A. 2017. Khan Forest protected area systems and biodiversity conservation in Bangladesh. In: S. A. Mukul and A. Z. M. M. Rashid (eds.) Protected Areas: Policies, Management and Future Directions. Nova Science Publishers, USA. pp. 157-177.

Mukul, S. A. 2007. Biodiversity conservation strategies in Bangladesh: the state of protected areas. Tiger paper. 34: 28-32.

Nishat, A., S. M. I. Huq, S. P. Barua, A. H. M. A. Reza and A. S. M. Khan. 2002. Bio-ecological Zones of Bangladesh. Dhaka. The World Conservation Union.

Richardson, D. M., J. J. Hellman, J. S. McLachlan, D. F. Sax and others. 2009. Multidimensional evaluation of managed relocation. Proc. Natl. Acad. Sci. USA. 106: 9721-9724.

Sarasan, V., R. Cripps, M. M. Ramsay, C. Atherton, M. McMichen, G. Prendergast and J. K. Rowntree.2006. Conservation in vitro of threatened plants-progress in the past decade. In Vitro Cell Dev. Biol. Plant. 42: 206-214.

Siddiqui, K. U., M. A. Islam, Z. U. Ahmed, Z. N. T. Begum, M. A. Hassan, M. Khondker, M. M. Rahman, S. M. H. Kabir, M. Ahmad, A. T. A. Ahmed, A. K. A. Rahman and E. U. Haque (eds). 2007a. Encyclopedia of Flora and Fauna of Bangladesh. Bryophytes, Pteridophytes and Gymnosperms. Vol. 5. Asiatic Society of Bangladesh, Dhaka, pp. 1-391.

Siddiqui, K. U., M. A. Islam, Z. U. Ahmed, Z. N. T. Begum, M. A. Hassan, M. Khondker, M. M. Rahman, S. M. H. Kabir, M. Ahmad, A. T. A. Ahmed, A. K. A. Rahman and E. U. Haque (eds). $2007 \mathrm{~b}$. Encyclopedia of Flora and Fauna of Bangladesh. Angiosperms: Monocotyledons (AgavaceaeNajadaceae). Vol. 11. Asiatic Society of Bangladesh, Dhaka. 399 pp.

Smith R. D., J. B. Dickie, S. H. Linington, H. W. Pritchard and R. J. Probert (eds). 2003. Seed conservation: turning science into practice. Kew Publishing, London. 1023 pp.

TEEB (The Economics of Ecosystems and Biodiversity). 2010. The economics of ecosystems and biodiversity: mainstreaming the economics of nature; a synthesis of the approach, conclusions and recommendations of TEEB. Progress Press, Malta. 429 pp. 